\title{
Bio-sketches: An iterative process that allows recognizing, extraction, and adaptation of the essential characteristics of biological models
}

\author{
Keywords \\ Bio-sketches, Biological models, Design, Iterative process, Levels of abstraction.
}

One of the biggest challenges that first-year design studio students experience in a traditional ideation process is when they have to face the blank page to begin drawing ideas after reading the brief instructions. It was in 2020 , that as a teacher I looked for pedagogical alternatives that will generate a lower sense of frustration in students, that's why I started experimenting with an induction approach based on the essential features of biological models (Gebehuber \& Drack, 2008), an interdisciplinary approach that allows combining design and biology methods with the purpose that students acquire an ability to recognize and understand structures of nature that facilitate the drawing of concepts and forms for their first sketches. All starts from some already existing biological solutions, from which a useful principle is extracted at any of its scales (cell, tissue, organ, system) with which Nature has managed to complete a task (Wiltschnig et al. 2013), and subsequently, a search process for a design problem is carried out to which this principle can be applied with accuracy. This drawing exercise was a valuable option, especially for the moment of virtual education in design that we experienced last year, managing to improve the ideation process that we carried out in class through the screen. I name this exercise bio-sketches, and it has the goal that students can refine structural patterns of nature through a cyclical process of drawing sketches. Later in 2021, already with greater experience and knowledge of the methodology,
I wanted to enhance that purpose by developing an incremental iteration model with three stages where different levels of thought are developed, getting students to draw sketches with high levels of innovation. Based on some reference texts (Benyus, 1997; Arciszewski \& Cornell, 2006; Nagel et al., 2018), the iterative abstraction process proposal is characterized in the following way: The first loop of iteration of bio-sketches has a low level of abstraction, where the drawings are elementary and are reduced to imitating the shape directly and superficially using reference images. In nature, it is found as an evolutionary strategy known as mimicry, which by copying some characteristics increases the chances of survival. It includes the study of categories from morphology in terms of the shape and geometry of living organisms. The second loop of iteration of bio-sketches has a medium level of abstraction, it studies the processes in context, as a state of changes in the physical environment in which a series of interrelating elements, in nature it is found as the biological principle of adaptation. It includes the study of categories of behavior in terms of response processes to stimuli. The third loop of iteration of bio-sketches has a high level of abstraction that seeks new knowledge from a deep understanding, to imitate the systems, principles, and strategies or patterns, which in nature is found as the biological principle of mutation. It includes the study of categories of physiology in terms of functions of living systems, subsystems, and their parts. 